\title{
Solution of Radiation Problems Using the Fast Multipole Method ${ }^{\dagger}$
}

\author{
İBRAHIM KüRŞAT ŞENDUR AND LEVENT GÜREL* \\ DEPARTMENT OF ELECTRICAL AND EleCTronics ENGINEERING \\ BILKENT UNIVERSITY \\ BilKent, ANKARA, TURKey \\ (lgurel@ee.bilkent.edu.tr)
}

\section{Introduction}

In this work, electromagnetic radiation problems involving electrically large radiators and reflectors are to be solved using the fast multipole method (FMM). Structures that are intentional radiators, such as antennas, have to be designed to optimize the required radiation characteristics, such as the field pattern, directivity, impedance, and bandwidth. On the other hand, unintentional radiators, such as electronic systems, have to be designed to supress the radiation mechanisms.

Computational simulation of both types of radiators can be used to improve the design in a shorter time spending less resources. However, some interesting real-life radiation problems are electrically very large and thus cannot be solved using traditional solution algorithms due to the limitations on the computational resources. The FMM [1-3] enables the solution of larger problems with existing computational resources by reducing the computational complexity and the memory requirement of the solution without sacrificing the accuracy. This is achieved by replacing the matrix-vector multiplications of $O\left(N^{2}\right)$ complexity by a faster equivalent of $O\left(N^{1.5}\right)$ complexity in each iteration of an iterative scheme. Some versions of the FMM have complexities that are even lower than $O\left(N^{1.5}\right)$ per iteration. In contrast, a direct solution would require $O\left(N^{3}\right)$ operations.

$3 \mathrm{D}$ radiation problems involving complicated geometries will be modelled using arbitrary surface triangulations. Piecewise linear basis functions defined on triangular domains due to Rao, Wilton, and Glisson [4] and referred to as RWG basis functions will be used to approximate the induced currents.

\section{Source Types}

For the excitation of radiation systems, numerous different source types can be used. Examples are Hertzian electric dipoles, delta-gap voltage sources, and finite-sized current distributions. The Hertzian electric dipole is a current element with an infinitesimal length. It is the simplest of all radiating systems. Delta-gap voltage sources can be

\footnotetext{
'This work was supported in part by NATO's Scientific Affairs Division in the framework of the Science for Stability Programme and in part by the Scientific and Technical Research Council of Turkey (TUBITAK) under contract EEEAG-163.
}

0-7803-4178-3/97/\$10.00 (C) $1997 \mathrm{IEEE}$ 
treated as generators of constant electric field in infinitesimal regions. Source current distributions of finite size can be defined on conducting structures using subdomain functions similar to the basis functions. For instance, if RWG basis functions are used to model the current on a conducting surface that is represented by a triangulation, then the source distrribution can also be modelled by RWG functions with known amplitudes. Half-RWG functions, full RWG functions or a combination of them can be used to represent the source current distribution. Electromagnetic fields in the source region may become singular and the numerical computation of radiation integrals must be handled carefully.

\section{A Sample Problem}

A Hertzian electric dipole radiates in the presence of a perfectly conducting sphere as shown in Fig. 1. The dipole is placed in the radial direction with respect to the center of the sphere. The analytical solution of this problem is given by Harrington [5]. $E_{\theta}$ for

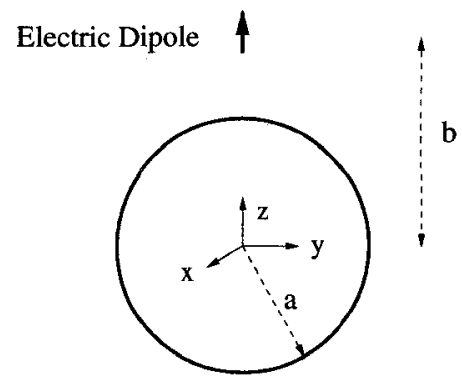

Figure 1: Radiation from a Hertzian electric dipole in the presence of a sphere.

$\phi=0$ in the far-field region is given by

$$
E_{\theta}=\frac{\omega \mu}{4 \pi} \frac{e^{i k r}}{r} I l \frac{E_{0}}{k^{2} b^{2}} \sum_{n=1}^{\infty} n(n+1)\left[a_{n} \hat{J}_{n}(k b)+b_{n} \hat{H}_{n}^{(1)}(k b)\right](-1)^{n} P_{n}^{1}(\cos \theta),
$$

where

$$
a_{n}=\frac{i^{n}(2 n+1)}{n(n+1)}, \quad b_{n}=-a_{n} \frac{\hat{J}_{n}^{\prime}(k a)}{\hat{H}_{n}^{(1)}(k a)}, \quad \text { and } \quad \hat{B}_{n}(x)=\sqrt{\frac{\pi x}{2}} B_{n+\frac{1}{2}}(x) .
$$

This solution will be used as a reference solution to check our computational results obtained by the FMM and MoM solutions of a surface integral equation. The computational solution requires the evaluation of the inner product of the testing function and the incident field, $\left\langle\mathbf{E}(\mathbf{r}), \mathbf{t}_{i}(\mathbf{r})\right\rangle$. In this problem the incident field is the electric field due to a Hertzian dipole and is given by

$$
\begin{aligned}
\mathbf{E}(\mathbf{r}) & =i \omega \mu\left[I+\frac{1}{k^{2}} \nabla \nabla\right] \cdot \int d V^{\prime} \frac{e^{i k\left|\mathbf{r}-\mathbf{r}^{\prime}\right|}}{4 \pi\left|\mathbf{r}-\mathbf{r}^{\prime}\right|} \hat{z} I l \delta\left(\mathbf{r}^{\prime}\right) \\
& =-i \omega \mu I l \frac{e^{i k r}}{4 \pi r}\left\{\hat{r}\left[\frac{i}{k r}+\left(\frac{i}{k r}\right)^{2}\right] 2 \cos \theta+\hat{\theta}\left[1+\frac{i}{k r}+\left(\frac{i}{k r}\right)^{2}\right] \sin \theta\right\} .
\end{aligned}
$$




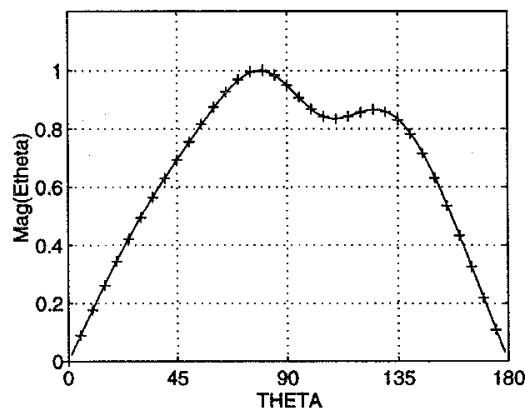

(a)

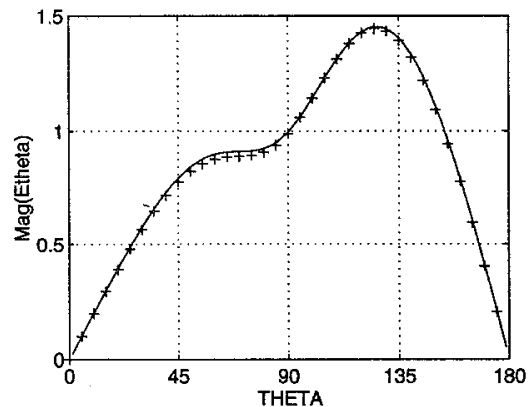

(c)

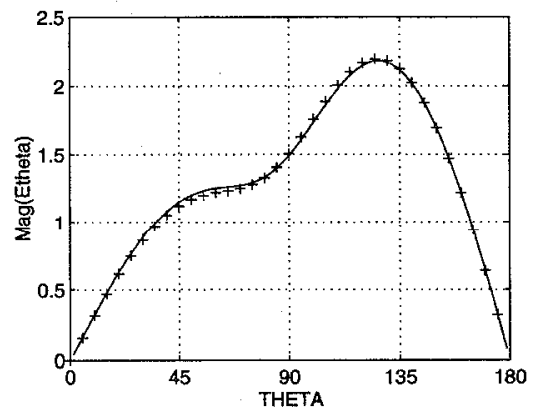

(e)

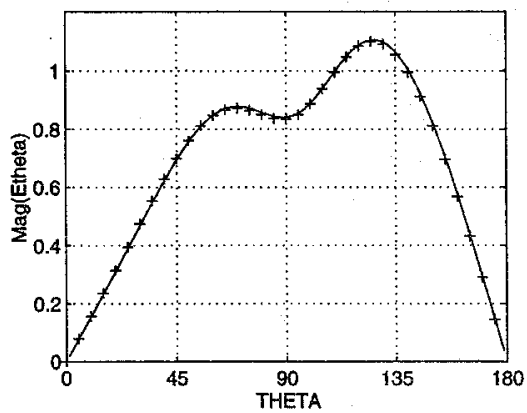

(b)

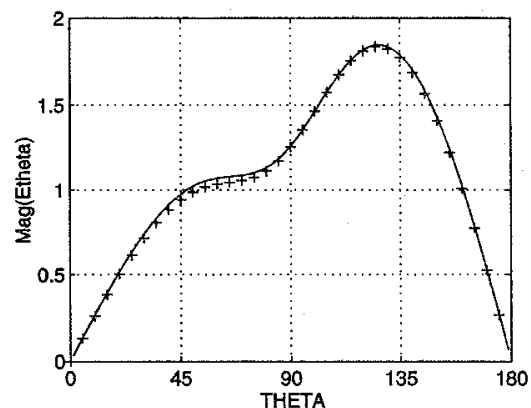

(d)

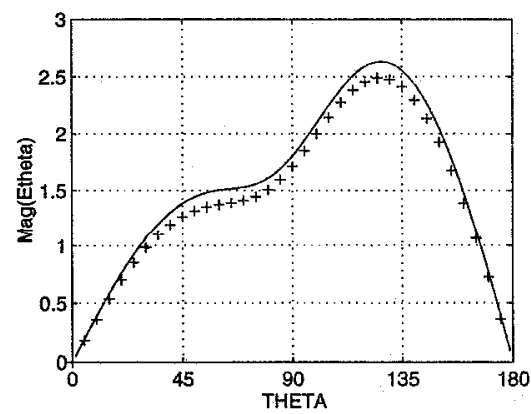

(f)

Figure 2: Analytical ( - and numerical ( + ) results for $E_{\theta}$ on $\phi=0$ cut for different values of $b:$ (a) $b=a+\lambda / 2$. (b) $b=a+\lambda / 4$. (c) $b=a+\lambda / 8$. (d) $b=a+\lambda / 16$. (e) $b=a+\lambda / 32$. (f) $b=a+\lambda / 256$. 
If the dipole is close to the sphere, the singularity of the dipole field renders the numerical computation of the inner product difficult. In that case, a singularity-extraction technique has to be used [6].

As a test case, the radius of the sphere is chosen to be $a=\lambda / 4$. The induced currents on the sphere is represented by 480 RWG basis functions. As the distance of the dipole to the sphere center, $b$, changes from $a+\lambda / 2$ to $a+\lambda / 256$, the $\theta$ component of the total electric field on the $\phi=0$ cut is computed and displayed in Fig. 2. The analytical and computational results are in good agreement in Figs. 2(a) and (b), where the dipole is placed at a distance of $\lambda / 2$ and $\lambda / 4$, respectively, from the surface of the sphere. When the dipole gets closer to the sphere, the induced current on the surface of the sphere is expected to change rapidly near the north pole, and hence the discretization becomes insufficient. The analytical and computational results start deviating from each other as shown in Figs. 2(c)-(f).

\section{Applications}

By implementing the source types given in Section 2 in an FMM formalism, we are planning to solve some interesting radiation problems. One such problem is obtained by placing a source in a conducting box with apertures. This problem can be used to model radiation from system enclosures with openings, which is an important issue in electromagnetic compatibility (EMC). By solving this problem at a number of different operating frequencies, we can investigate the effects of the box resonances on the radiation from the apertures.

Using delta-gap voltage sources and prescribed current distributions, we can simulate the operations of various antennas and study their radiation characteristics as a function of frequency. Spurious radiation and crosstalk in printed-circuit-board (PCB) and monolithic-microwave-integrated-circuit (MMIC) structures can also be studied by employing these sources and exploiting the power of the FMM to solve large problems. Progress on these issues will be reported.

\section{References}

[1] V. Rokhlin, "Rapid solution of integral equations of scattering theory in two dimensions," $J$. Comput. Phys., vol. 86, pp. 414-439, Feb. 1990

[2] R. Coifman, V. Rokhlin, and S. Wandzura, "The fast multipole method for the wave equation: a pedestrian prescription," IEEE Antennas Propagat. Mag., vol. 35, no. 3, pp. 7-12, June 1993.

[3] C. C. Lu and W. C. Chew, "Fast algorithm for solving hybrid integral equations,' Proc. IEE, vol. 140, Part H, pp. 455-460, Dec. 1993.

[4] S. M. Rao, D. R. Wilton, and A.W. Glisson, "Electromagnetic Scattering by surfaces of arbitrary Shape," IEEE Antennas Propagat. Mag., vol. 30, no. 3, pp. 409-418, May 1982.

[5] R. F. Harrington, Time-Harmonic Electromagnetic Fields. Piscataway, NJ: IEEE Press, 1994.

[6] R. D. Graglia, "On the numerical integration of the linear shape functions times the 3-D Green's function or its gradient on a plane triangle," IEEE Antennas Propagat. Mag., vol. 30, no. 3, pp. 409418, May 1982. 